\title{
LISA Telescope Assembly Optical Stability Characterization for ESA
}

\author{
Adrianus L. Verlaan ${ }^{\mathrm{a}}$, Harmen Hogenhuis ${ }^{\mathrm{a}}$, Joep Pijnenburg ${ }^{\mathrm{a}}$, Martin Lemmen ${ }^{\mathrm{a}}$, Stefano Lucarelli ${ }^{\mathrm{b}}$, \\ Dietmar Scheulen ${ }^{b}$, David Ende ${ }^{c}$ \\ a. TNO, Stieltjesweg 1, Postbus 155 2600AD Delft, The Netherlands \\ b. EADS ASTRIUM, 88039 Friedrichshafen, Germany \\ c. xperion AEROSPACE GmbH, Claude-Dornier-Str., 88090 Immenstaad Germany,
}

\begin{abstract}
The LISA Optical Stability Characterization project is part of the LISA CTP activities to achieve the required Technonlogy Readiness Level (TRL) for all of the LISA technologies used. This activity aims demonstration of the Telescope Assembly (TA), with a structure based on CFRP technology, that a CTE of $10^{-7} 1 / \mathrm{K}$ can be achieved with measures to tune the CTE to this level. In addition the demonstration is required to prove that the structure exhibits highly predictable mechanical distortion characteristics when cooling down to $-90^{\circ} \mathrm{C}$, during outgassing in space and when going from $1 \mathrm{~g}$ environment to $0 \mathrm{~g}$.

This paper describes the test facilities as well as the first test results. A dedicated test setup is designed and realized to allow monitoring dimensional variations of the TA using three interferometers, while varying the temperature in a thermal vacuum chamber. Critical parameters of the verification setup are the length metrology accuracy in thermal vacuum and the thermal vacuum flexibility and stability. The test programme includes Telescope Assembly CTE measurements and thermal gradient characterization.
\end{abstract}

Keywords: LISA, NGO, telescope, characterization

\section{INTRODUCTION}

The primary goal of the Laser Interferometer Space Antenna (LISA) mission is the detection of gravitational waves from astronomical sources in a frequency range of $10^{-4}$ to $1 \mathrm{~Hz}$. This requires operational stabilities in the picometer range as well as highly predictable mechanical distortions upon cooling down, outgassing in space, and gravity release. In March 2011 ESA announced a new way forward for the L-class candidate missions, including LISA. ESA and the scientific community are now studying options for European-only missions that offer a significant reduction of the costs, while maintaining their core science objectives. In the context of this reformulation exercise LISA has become the New Gravitational wave Observatory (NGO) [1].

Despite this reformulation, the need for dimensional stability in the picometer range remains valid, and ESA have continued the corresponding LISA Technology Development Activities (TDA's) also in view of NGO. In such frame an elegant breadboard of the LISA Telescope Assembly (TA) structure is designed and will be tested in a newly developed test facility. Astrium GmbH and xperion (Immenstaad/Friedrichshafen, Germany) have designed and manufactured an ultra-stable CFRP breadboard of the LISA telescope in order to experimentally demonstrate that the structure and the M1 \& M2 mirror mounts are fulfilling the LISA requirements in the mission operational thermal environment. Suitable techniques to mount the telescope mirrors and to support the M1 \& M2 mirrors have been developed, with the aim of measuring a system CTE of less than $10^{-7} \mathrm{~K}^{-1}$ during cooling down to $-90{ }^{\circ} \mathrm{C}$. Additionally to the stringent mass and stiffness specifications, the required offset design makes the control of relative tilts and lateral displacements between the M1 and M2 mirrors particularly demanding.

The thermo-mechanical performance of the telescope assembly is going to be experimentally verified by TNO (Delft, The Netherlands) starting from the second half of 2012. For the purpose of these experiments a complex verification setup is designed and realized, which comprises of a dedicated Thermal Vacuum Chamber (TVC) equipped with three displacement interferometers. These interferometers monitor length changes directly between the TA-structure mirrors, providing mirror relative displacement and tilt information, while the TA-structure is exposed to a thermal cycle. The test programme includes Telescope Assembly CTE measurements and thermal gradient characterization.

This paper addresses challenges faced in the TA-structure design phase, and shows the resulting hardware. Furthermore the realized test facility design and operation are explained. The paper is concluded by the first measurement results. 


\section{TELESCOPE ASSEMBLY STRUCTURE}

The TA-structure is designed by Astrium Gmbh, based on components delivered by project partners. Carbon Fibre Reinforced Plastic (CFRP) and metal parts are realized by Xperion and the zerodur mirror blanks by Schott. TNO has grinded and polished the test mirrors to their final shape and provided them with the required coatings. To simulate the interface to the LISA Optical bench a high stability Invar structure is designed and realized. In figure 1 the TA-structure and the test mirrors are shown during the integration at Astrium.
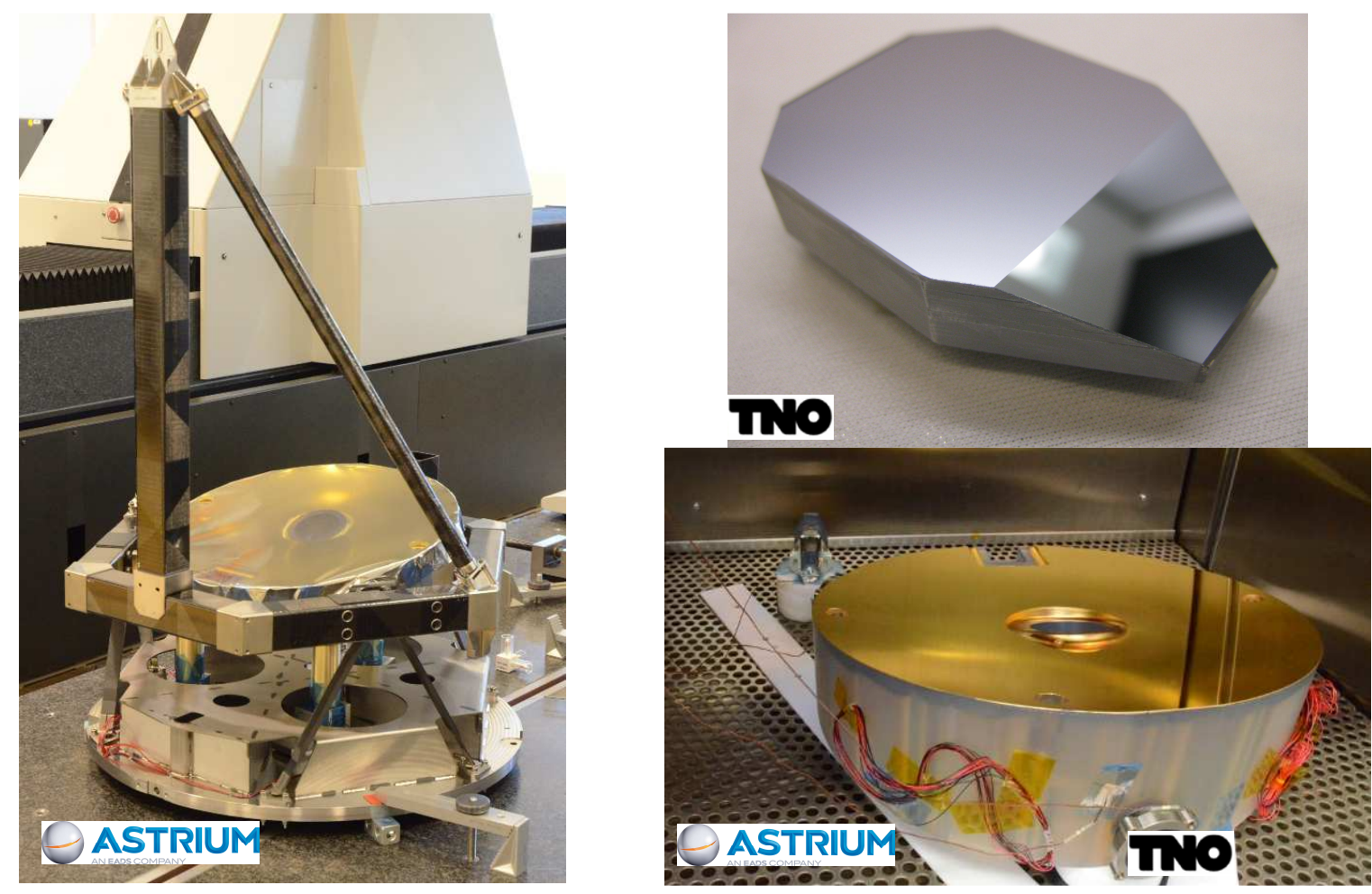

Figure 1 Left: Telescope Assembly structure at Astrium under the coordinate measurement machine. Right top: Secondary test mirror. Right bottom: Primary test mirror after gluing of inserts.

\begin{tabular}{|l|l|l|}
\hline Specification & Requirement & Predicted results \\
\hline Mass, without mirrors & $<5.5 \mathrm{~kg}$ & $4.8 \mathrm{~kg}$ \\
\hline First eigenfrequency & $>80 \mathrm{~Hz}$ & $82 \mathrm{~Hz}$ \\
\hline Gravity & & \\
M1 surface distorsion & $<10 \mathrm{~nm} \mathrm{rms}$ & $5.7 \mathrm{~nm} \mathrm{rms}$ \\
\hline$\underline{\Delta T=100 K}$ & & \\
Distance M1-M2 & $<6 \mu \mathrm{m}$ & $2.1 \mu \mathrm{m}$ \\
Rel. lat. displ. M2-M1 & $<2 \mu \mathrm{m}$ & $0.7 \mu \mathrm{m}$ \\
Rel. tilt M2-M1 & $<20 \mu \mathrm{rad}$ & $8.2 \mu \mathrm{rad}$ \\
\hline 18K axial T-gradient & & \\
Distance M1-M2 & $<6 \mu \mathrm{m}$ & $0.4 \mu \mathrm{m}$ \\
Rel. lat. displ. M2-M1 & $<2 \mu \mathrm{m}$ & $0.6 \mu \mathrm{m}$ \\
Rel. tilt M2-M1 & $<20 \mu \mathrm{rad}$ & $1.5 \mu \mathrm{rad}$ \\
\hline Moisture desorption & & \\
Distance M1-M2 & $<6 \mu \mathrm{m}$ & $0.1 \mu \mathrm{m}$ \\
Rel. lat. displ. M2-M1 & $<2 \mu \mathrm{m}$ & $\sim 0 \mu \mathrm{m}$ \\
Rel. tilt M2-M1 & $<20 \mu \mathrm{rad}$ & $4.7 \mu \mathrm{rad}$ \\
\hline
\end{tabular}

Table 1: Telescope Assembly structure design properties and predicted deformation due to the different load cases. 
Core element of the TA-structure is the CFRP, which is light yet stiff and can be designed to the required CTE. By carefully analyzing all predicted load cases, the structure is designed to yield a very small $\left(<10^{-7} 1 / \mathrm{K}\right) \mathrm{CTE}$ around operational temperatures ( -45 to $-90 \mathrm{DegC}$ ) and low deformation during the cooldown. Design choices are supported by material experiments and extensive FEM analyses [2]. The table above summarizes the main results obtained from the finite element predictions at the end of the detailed design phase of the truss design concept.

\section{THERMAL VACUUM CHAMBER}

A significant part of the studies is addressed to the characterization and demonstration of the TA-structure thermal behaviour. A newly developed Thermal Vacuum Chamber is designed to offer a combination of high thermal stability $(<<0.1 \mathrm{~K} /$ hour $)$ and uniformity $(<1 \mathrm{~K})$ as well as fast thermal adjustment. In the TVC design more stringent thermal stabilities are anticipated to allow future use for direct demonstration of pm-stabilities. In figure 2 the TVC design and realized setup are shown.
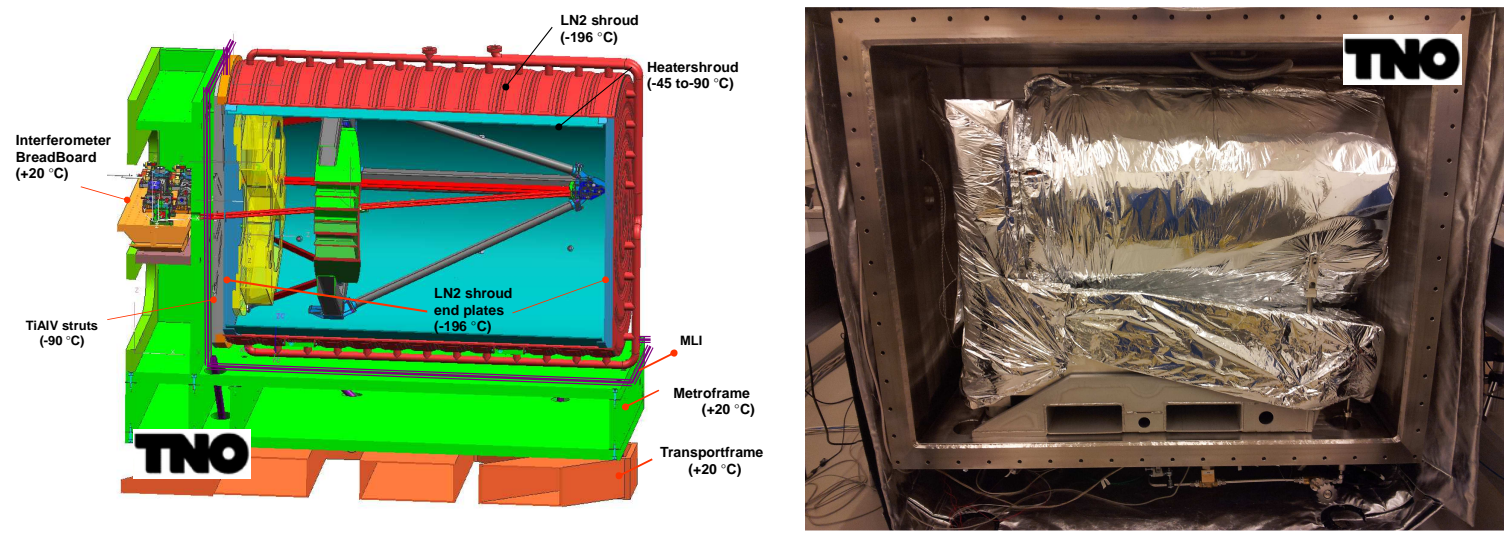

Figure 2 Schematic representation of the Thermal Vacuum Facility at TNO on the left and the realized setup on the right.

Two separate thermal zones are created in the TVC, a warm vacuum and the thermal vacuum. The warm vacuum contains the support frame (green) and the interferometer breadboard (orange) and is maintained at a temperature slightly above ambient. This way the metrology performance and stability can be guaranteed the best. By thermally insulating the interferometer (IFM) breadboard from the surrounding support frame using MLI-blankets, predicted temperate variations at this location are significantly smaller than $0.1 \mathrm{~K}$.

The thermal vacuum contains the TA-structure and is primarily designed to create a stable and homogenous thermal environment. This is achieved using a combination of two thermal shrouds. A liquid Nitrogen shroud (in red) isolates the inner part of the thermal vacuum from all thermal variations in the laboratory and is used as a stable thermal basis, without introducing mechanical vibrations. An inner shroud is electrically heated to any required temperature between 100 and +60 DegC. The Lisa telescope assembly breadboard is suspended at the same temperature within this environment thermally fully decoupled from the metroframe, using titanium struts and copper straps, resulting in a temperature uniformity of $<0.1$ DegC. Extensive analyses are performed to support the design. In figure 3 some simulation results are given on the impact of the heater wire pattern. For the electrical shroud an overall homogeneity of better than $1 \mathrm{~K}$ and a stability below $0.1 \mathrm{~K}$ are predicted. 

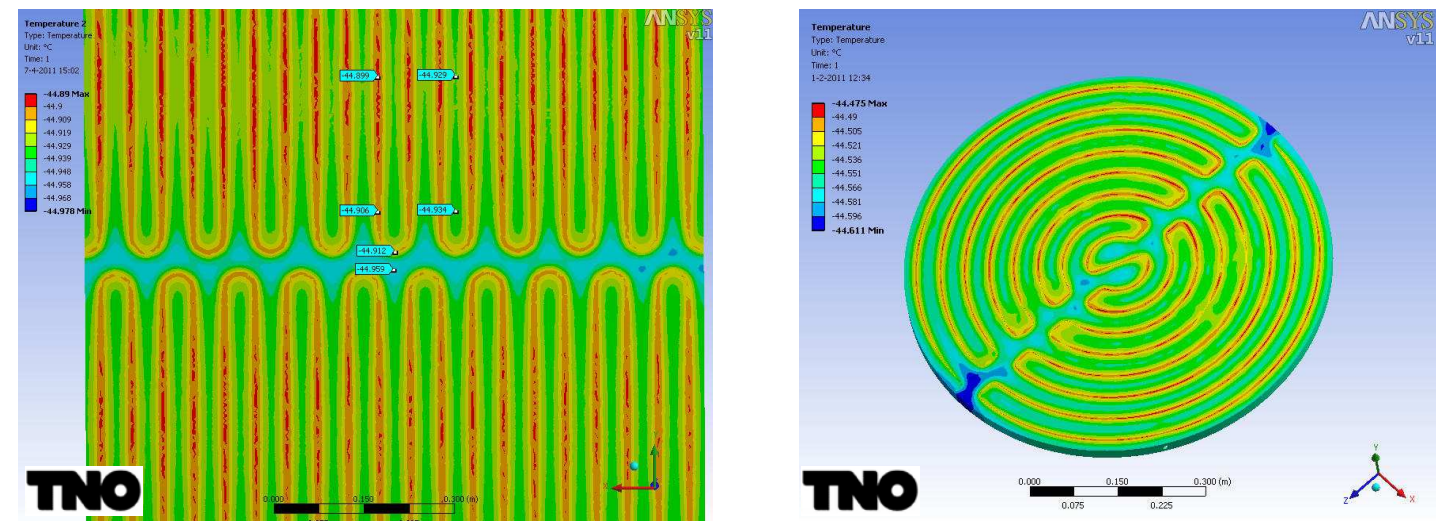

Figure 3: Electrical shroud temperature distribution due to heater wiring pattern around $-45 \mathrm{DegC}$ for barrel (60mK on left) and endplate ( $14 \mathrm{mK}$ on the right).

A second operational mode of the TVC is to include the impact of partial exposure of the TA-structure to cold space. To simulate cold space the electrical shroud end plate (on the right) can be switched off (or removed), reducing its temperature significantly. As a result a thermal gradient along the TA-structure of $18 \mathrm{~K}$ is predicted.

For the TVC thermal control heaters and sensors are placed on separate parts of the facility as well as on the TAstructure. Overall 14 thermal control loops and 83 sensors are used. The actual vacuum is created by a combination of a $40 \mathrm{~m}^{3} / \mathrm{hr}$ pre-vacuum pump and a 30001/s turbo-pump. The combination of these relatively large pumps allows reaching $10^{-6} \mathrm{mbar}$ pressures in a few hours, despite the large amount of MLI blanket in the facility. For outgassing and contamination monitoring the TVC is equipped with a Rest Gas Analyser (RGA) and witness samples are used. Possible impact of vibrations to the length measurements are reduced in three steps. First of all the stiffness of the setup is made large, so that the received vibrations result in common mode motion of the setup, not affecting the length measurement directly. Secondly the entire TVC is placed on vibration isolators, which reduce the vibration amplitude above $1 \mathrm{~Hz}$. Inside the TVC a second stage of vibration isolation is included, which holds the metrology frame and the TA-structure. The thermal shrouds are mounted separately onto the transport frame, reducing the transfer from the potential liquid nitrogen shroud vibrations to the metrology frame.

\section{LENGTH METROLOGY SYSTEM}

The length metrology system consists of three displacement interferometers to measure the deformations of the breadboard telescope through the thermal vacuum experiments, see figure 4. Using these three relative displacement interferometers the lateral and longitudinal displacement between the test mirrors are monitored optical directly.

Lab.

Warm vacuum Thermal Vacuum

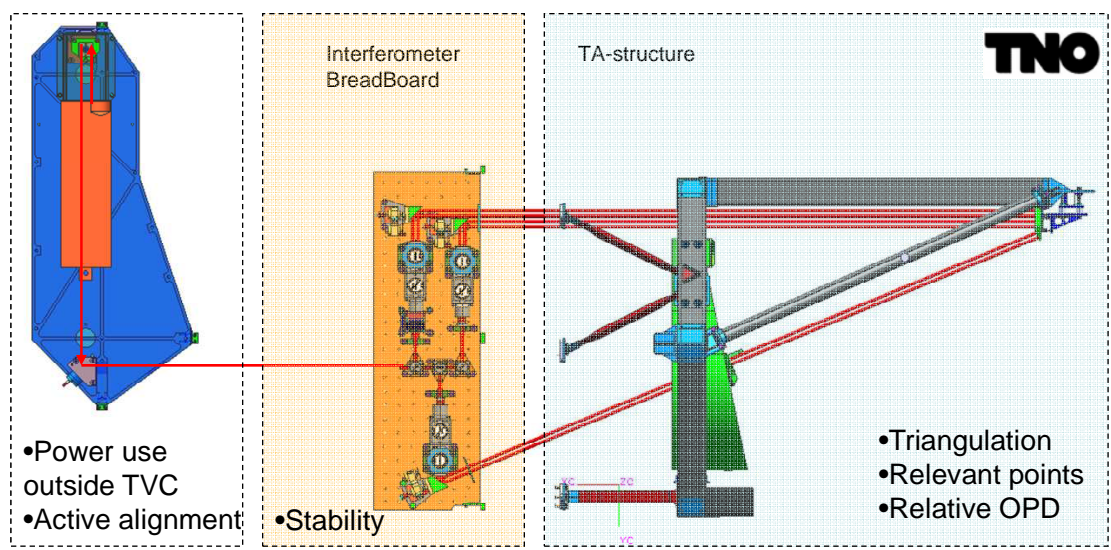

Figure 4 Schematic overview of the length metrology, showing: Laser source outside the vacuum with active beam alignment, high stability IFM bench in the warm vacuum and the TA-structure in the thermal vacuum 
The actual IFM light source, the laser, as well as the detectors are located outside the TVC. This way commercial devices can be used and the main (un-controlled) heat sources are removed from the TVC. In order to allow for correction of the beam pointing two tip-tilt mirrors are included in the optical path. The laser beam is pointed towards the IFM breadboard through a vacuum window in the TVC.

The IFM's are placed on an aluminium breadboard in the warm vacuum. The incoming beam is distributed over the three channels using beam splitters. Each channel contains a dual pass differential IFM head (Zygo) and a high stability steering mirror.
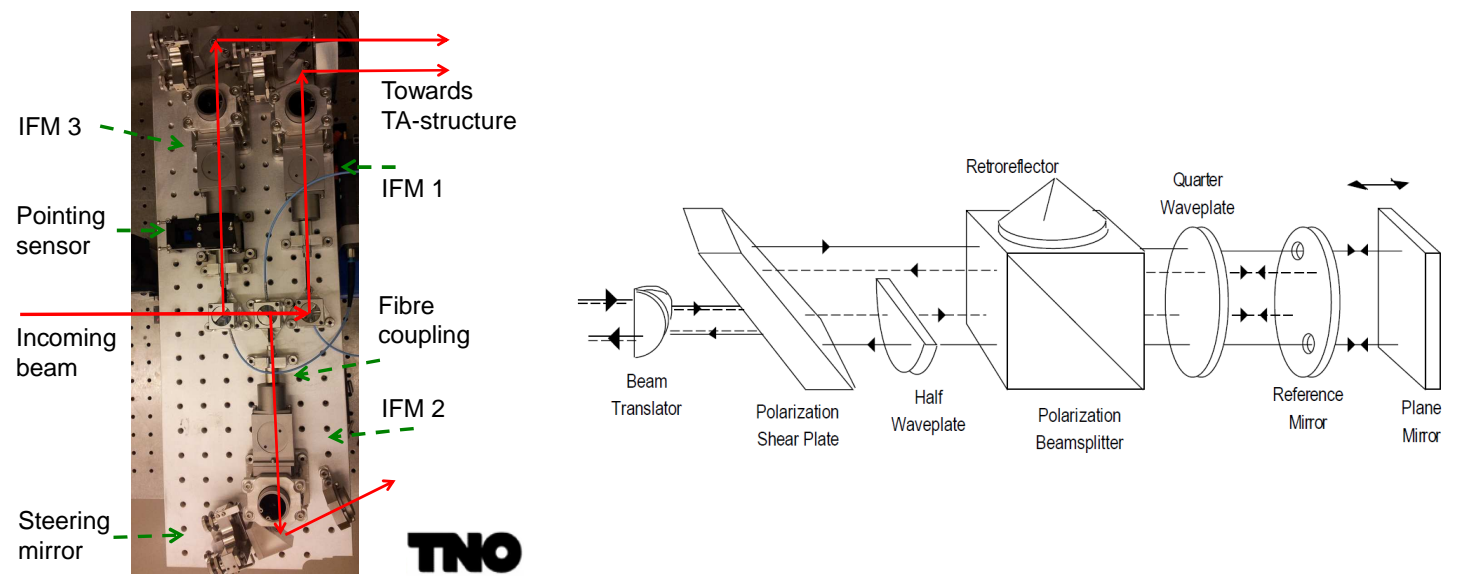

Figure 5 Picture of the interferometer bench the warm vacuum during laboratory testing (left) and schematic build-up of a single interferometer (right)

In order to meet the TA-structures' deformation requirements the metrology system should measure length changes with an accuracy better than $70 \mathrm{~nm}$ over a few hours and $200 \mathrm{~nm}$ over the full duration of the experiments. This can be as long as a week. Obviously the sensitivity of the metrology will be in the nanometer range. However in these experiments the lonh term performance is the most critical.

An essential property of the selected IFM is that both the reference beam and measurement beam effectively measure at a common position, making the IFM insensitive to tilt in the first order $^{3}$. The main angular dependence that remains is the cosine angle. In order to keep this error contributor small (few tens of nanometers) a beam alignment system is included in the setup. The beam alignment is measured to the $\mu \mathrm{rad}$ level accuracy using the pointing sensor. Variations in the alignment are corrected actively with the tip-tilt mirrors outside the vacuum.

The TA-structure is located in the thermal vacuum and is exposed to a large operational temperature range (-90 to 45Deg C ). As a results it is important to measure the mirror displacement directly. Adding elements or interfaces complicates the distinction between the setup and measurement object. Alternative approaches for the measurement over the optical path can be thought of, however they often rely on the stability of a reference frame, which stability cannot be guaranteed in the thermal vacuum. Two metrology inserts are implemented in the test mirrors, since the zerodur is not sufficiently transparent and to combine the 2 functions in a single breadboard. Figure 6 shows how the inserts (red and orange) are mounted in the primary mirror (in green). The inserts are used in transmission to equalize the measurement and reference arms' thermal behaviour. Fort this purpose small reflective areas are coated onto one surface. Here the reference beams are reflected and the measurement beams transmitted. This way the monitored distance is the bare separation between the telescopes' primary and secondary mirrors. Using zerodur shims (blue) the inserts are mounted into the primary mirror with their front surface in the mirror plane. Two compression brackets (yellow) and springs are used to keep the inserts in place with a soft pre-tension. 

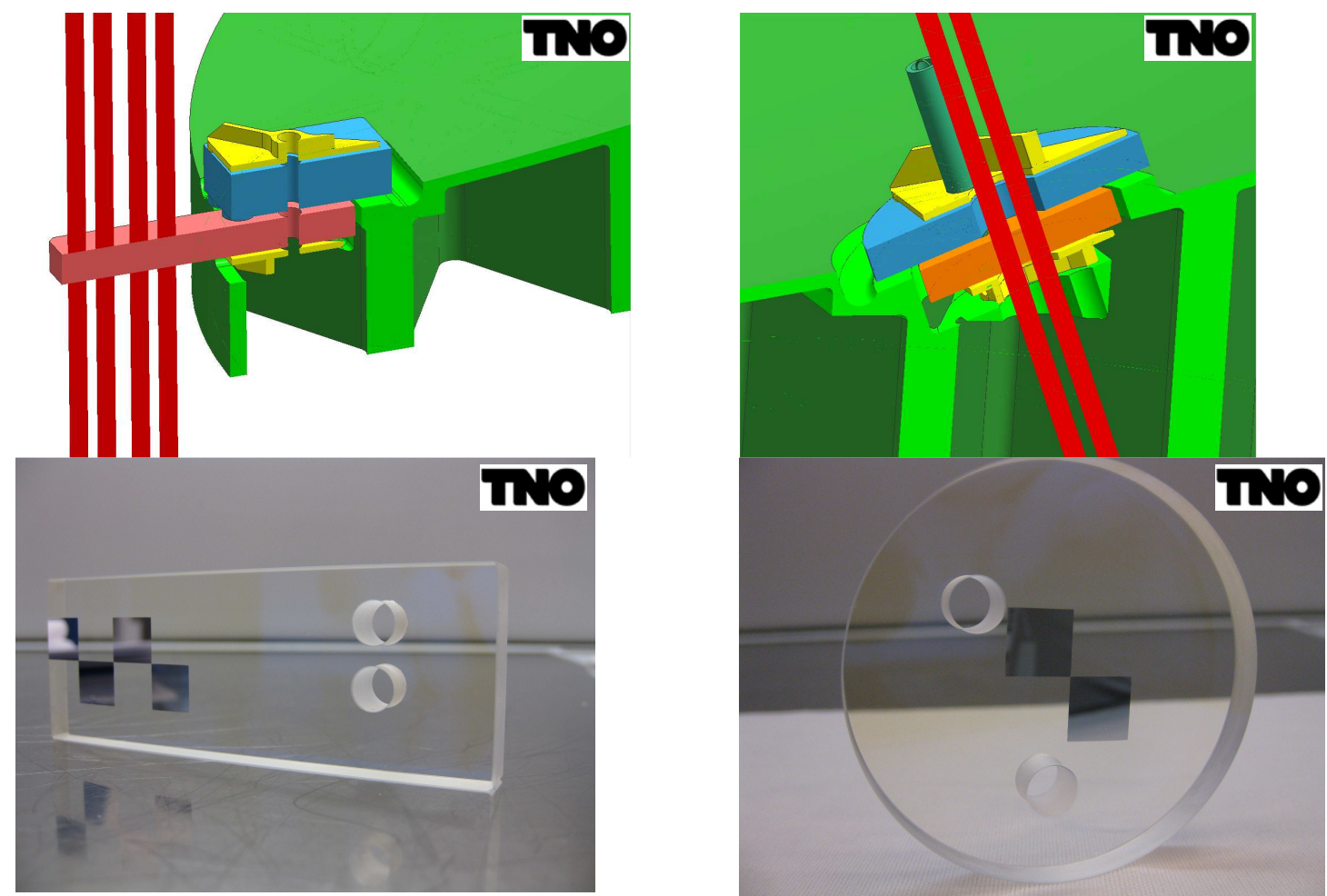

Figure 6 On the left: sketch and photo of metrology insert on the edge of the primary test mirror. On the right: Sketch and photo of the inserts in the primary mirror centre

\section{EXPERIMENTS}

Currently the Thermal Vacuum Chamber and the Length Metrology system are being integrated and tested together, on a dummy telescope. This dummy telescope will undergo a similar thermal trajectory as the breadboard model demonstrating the performance and limitations of the setup. These qualification experiments are planned to be finalized before the Test Readiness Review (TRR) in July 2012. In the period following the TRR the TA-structure will be tested extensively. Core of the test program comprises of a combined experiment in the Thermal Vacuum Chamber. In this experiment a thermal trajectory is following based on a number of stable temperatures and a thermal gradient configurations. From this experiment the CTE, integrated deformation and thermal gradient experiment are derived.

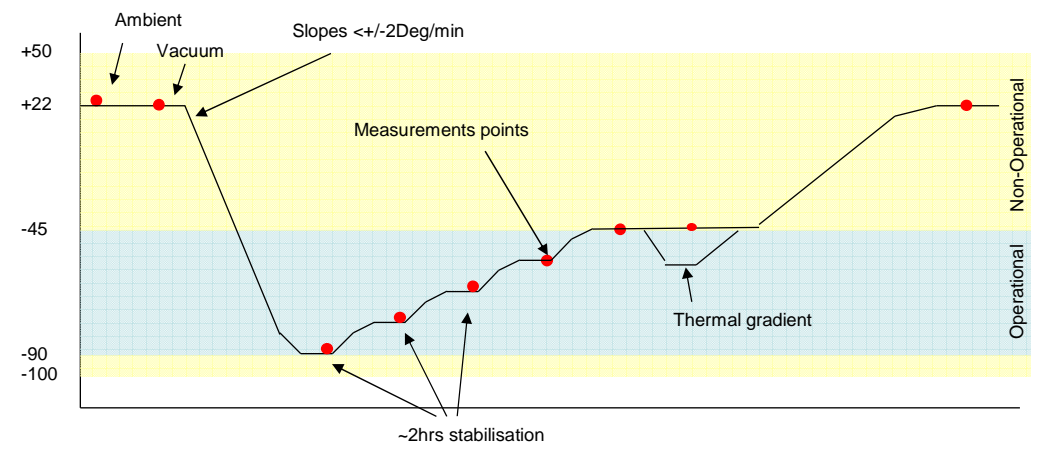

Figure 7 Combined thermal vacuum experiment, showing thermal trajectory and measurement points (bake-out phase not shown)

During the integration phase of the project the functionality of the TVC has been demonstrated. All sensors and control loops are operational and tested. In addition the liquid nitrogen system and the electrical heater shroud are operational. Here only final determination of the control parameters is required. 
The Interferometer bench has been tested separately in the lab to show stabilities of few tens of nm over a weekend. Since the thermal stability of the lab is far worse than in the TVC, the stability of the IFM signals is more than sufficient. In addition the beam pointing sensor was maintained operational over this period.

Currently the full integrated system is under test and the final control parameters are being determined. Measurements are performed using all included systems.

\section{CONCLUSIONS}

Although the experiments are on-going and important test results are to be acquired, a number of conclusions can already be drawn:

- A Telescope Assembly structure with challenging thermal stability properties has been designed, analysed and realized, aiming to demonstrate a CTE of less than $10^{-7} 1 / \mathrm{K}$ and a low and predictable distortion under a $18 \mathrm{~K}$ thermal gradient. The TA-structure is available at TNO for testing.

- For the verification of the challenging dimensional stability requirements a thermal vacuum chamber has been designed, realized and is undergoing its final qualification. First results on the commissioning show that the thermal vacuum chamber is operational.

- An extensive test campaign is put together, where the ultimate test of the facility (null test on a dummy telescope) is about to be started.

- First results on the commissioning show that the metrology design works and is sufficiently stable for the characterization of the telescope structure.

\section{ACKNOWLEDGMENTS}

Most of the work presented in this paper is performed under ESA contract ESTEC 4000101286/10/NL/HB: "Optomechanical Stability Characterisation for LISA". TNO is prime contractor for this project and have performed the activities in close cooperation with the sub-contractors EADS Astrium (Friedrichchshafen) and xperion:

\section{REFERENCES}

1. "NGO - Revealing a hidden Universe: opening a new chapter of discovery - Assessment Study Report", ESA/SRE(2011)19, December 2011

2. Stefano Lucarelli, Dietmar Scheulen, Daniel Kemper, Rudolf Sippel, David Ende, “THE BREADBOARD MODEL OF THE LISA TELESCOPE ASSEMBLY”, ESSMET, 2012.

3. "DISPLACEMENT MEASURING INTERFEROMETRY MEASUREMENT UNCERTAINTY", M. Holmes, C. Evans Zygo Corporation, Middlefield, CT 06455 